\title{
ANOTHER LOCALLY CONNECTED HAUSDORFF CONTINUUM NOT CONNECTED BY ORDERED CONTINUA
}

\author{
J. L. CORNETTE AND B. LEHMAN
}

Abstract. An example is given of a locally connected Hausdorff continuum which is not connected by ordered continua.

In 1960, S. Mardešić [2] gave an example of a locally connected Hausdorff continuum which is not connected by ordered continua (see also [3]). His example is somewhat complex, and it is the purpose of this paper to give a conceptually simpler example.

An ordered continuum is a totally ordered set $\{K,<\}$ such that $K$ with the topology induced by the total order is compact and connected. Every closed subset of an ordered continuum has a first point and a last point in the order; they share many of the properties of metric arcs, and may be characterized as being Hausdorff continua with only two noncut points. A space $X$ is said to be connected by ordered continua if for each two points $x$ and $y$ of $X$, there is an ordered continuum $K$ with first and last points $a$ and $b$ and a continuous map $f: K \rightarrow X$ such that $f(a)=x$ and $f(b)=y$.

We use the notation $(x, y),(x, y]$ and $[x, y]$ for nondegenerate open, half open and closed intervals of ordered continua, or of the real numbers when $x$ and $y$ are numbers, and the notation $\langle x, y\rangle,\langle x, y, z\rangle$ for ordered pairs and triples. Following Kelley [1], we let $\Omega_{0}$ denote the set of countable ordinals, $\Omega$ denote the first uncountable ordinal and $\Omega^{\prime}$ denote $\Omega_{0} \cup\{\Omega\}$. We let $L$ denote $\Omega_{0} \times[0,1) \cup\{\langle\Omega, 0\rangle\}$ with the order topology induced by the lexicographic order- $\langle p, q\rangle\langle\langle r, s\rangle$ if and only if $p<r$ or $p=r$ and $q<s$. Then $L$ is an ordered continuum, sometimes called "the long interval", with first point $\langle 0,0\rangle$ and last point $\langle\Omega, 0\rangle$.

1. The spaces $S_{0}$ and $S$. In the product space $C=L \times[0,1] \times[-1,1]$, let $S_{0}$ denote the closure of

$$
\{\langle\langle\alpha, t\rangle, y, z\rangle \in C \mid z=\operatorname{Sin} \pi /(1-t)\} .
$$

We suggest Figure 1 as a representation of $S_{0} . S_{0}$ is a continuum and is locally connected except at the points $\langle\langle\alpha, 0\rangle, y, z\rangle$ where $\alpha\rangle 0$.

Received by the editors December 7, 1971.

AMS 1970 subject classifications. Primary 54G20, 54F25, 54F05.

Key words and phrases. Locally connected, ordered continua, arcwise connected. 


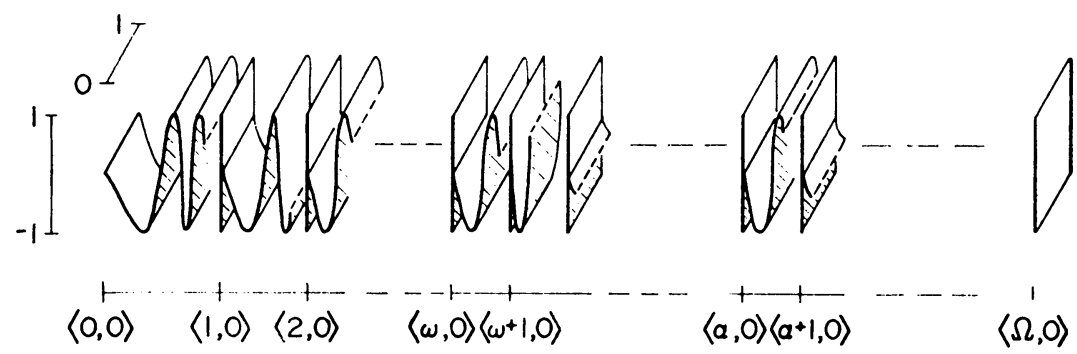

FIGURE 1

Let $\left\{\left\{\left\langle y_{\alpha, n}, z_{\alpha, n}\right\rangle\right\}_{n=1}^{\infty}\right\}_{\alpha \in \Omega_{0}}$ be a collection of countable dense subsets of $[0,1] \times[-1,1]$ with the property that $y_{\alpha, n}=y_{\bar{\alpha}, \bar{n}}$ implies that $\alpha=\bar{\alpha}$ and $n=\bar{n}$. For $\alpha$ in $\Omega_{0}$ and $n$ a positive integer, we let $L_{\alpha, n}$ be the interval in $C$ "parallel to $L "$.

$$
[\langle\alpha, 1-1 / n\rangle,\langle\alpha+1,0\rangle] \times\left\{y_{\alpha, n}\right\} \times\left\{z_{\alpha, n}\right\} .
$$

(See Figure 2.) We let

$$
S=S_{0} \cup \cup\left\{L_{\alpha, n} \mid \alpha \in \Omega_{0}, n=1,2, \cdots\right\} .
$$

Now $S$ is also a continuum; the effect of adding the intervals $\left\{L_{\alpha, n}\right\}$ to $S_{0}$ is that $S$ is locally connected at each point except the points $\langle\langle\alpha, 0\rangle, y, z\rangle$ where $\alpha$ is a limit ordinal. $S$ is locally connected at the nonlimit ordinals, $S_{0}$ is not. However, for each number $y_{1}$ in $[0,1]$, the "slice" $S_{y_{1}}=$ $\left\{\langle\langle\alpha, t\rangle, y, z\rangle \in S \mid y=y_{1}\right\}$ of $S$ contains at most one of the intervals $L_{\alpha, n}$, and is not locally connected at the points $\langle\langle\alpha, 0\rangle, y, z\rangle, \alpha\rangle 0$, except at an endpoint of perhaps that one interval.
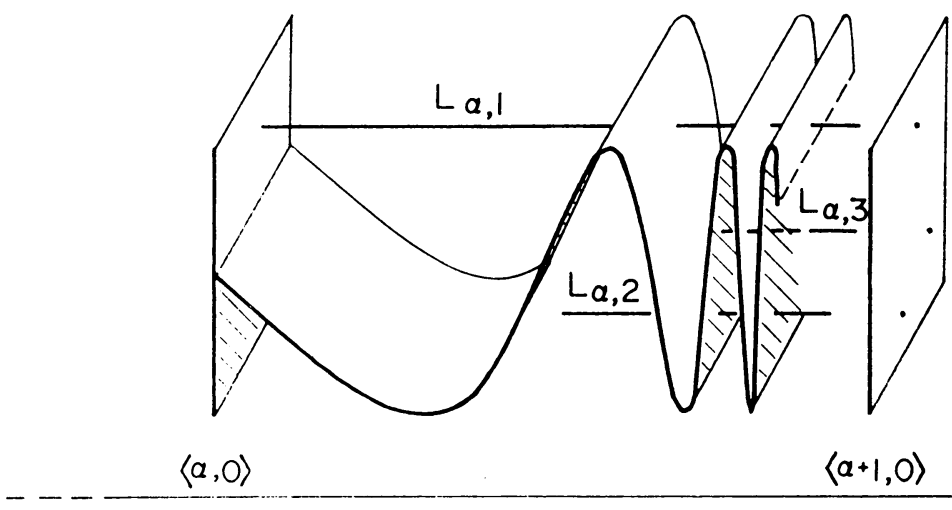

$\langle a+1,0\rangle$

FIGURE 2 
2. The example $M$. For each limit ordinal $\alpha$ in $\Omega^{\prime}$ and each number $y$ in $[0,1]$, let $V_{\alpha, y}$ denote the "vertical" interval

$$
V_{\alpha, y}=\{\langle\langle\alpha, 0\rangle, y, z\rangle \in S \mid-1 \leqq z \leqq 1\} .
$$

Let $M$ denote the upper semicontinuous decomposition of $S$ whose only nondegenerate elements are the intervals $V_{\alpha, y}$ where $\alpha$ is a limit ordinal and $y \in[0,1]$. ( $M$ is obtained from $S$ by identifying to a point each of the intervals $V_{\alpha, v}$.) Let $q: S \rightarrow M$ be the quotient map of the decomposition, For each member $P$ of $M$ and open set $O$ containing $P$, there is in $C$ a product open set $R=U \times V \times W, U$ open in $L, V$ open in $[0,1]$ and $W$ open in $[-1,1]$, such that $q(R \cap S)$ is a connected open set in $M$ which contains $P$ and is a subset of $O$. We omit discussion of the several special cases needed to establish this fact, but observe that with this and the fact that decompositions of continua yield continua, we have that $M$ is a locally connected Hausdorff continuum.

3. $M$ is not connected by ordered continua. Let $E_{\Omega}$ be $\left\{V_{\Omega, y} \in M \mid y \in\right.$ $[0,1]\}$. We show that $M$ is not connected by ordered continua between any point of $M-E_{\Omega}$ and any point of $E_{\Omega}$. The crux of the argument is:

LEMMA. If a subcontinuum $H$ of $T=L \times[0,1]$ contains only one point $\left\langle\langle\Omega, 0\rangle, y_{1}\right\rangle$ of $E=\{\langle\Omega, 0\rangle\} \times[0,1]$ and a point $\left\langle\left\langle\alpha_{0}, t_{0}\right\rangle, y_{0}\right\rangle$ of $T-E$, then there is a nondegenerate interval $L^{\prime}=\left(\left\langle\alpha^{\prime}, t^{\prime}\right\rangle,\langle\Omega, 0\rangle\right]$ of $L$ such that the intersection of $H$ with $L^{\prime} \times[0,1]$ is $L^{\prime} \times\left\{y_{1}\right\}$.

Proof. $E$ is homeomorphic to $[0,1]$, and there is a countable collection of open sets in $T, O_{i}=\left(\left\langle\alpha_{i}, t_{i}\right\rangle,\langle\Omega, 0\rangle\right] \times G_{i}, G_{i}$ open in $[0,1]-$ $\left\{y_{1}\right\}, i=1,2, \cdots$, such that $E-H \subset \bigcup_{i=1}^{\infty} O_{i} \subset T-H$. Let $\left\langle\alpha^{\prime}, t^{\prime}\right\rangle$ be the supremum in $L$ of $\left\{\left\langle\alpha_{0}, t_{0}\right\rangle\right\} \cup\left\{\left\langle\alpha_{i}, t_{i}\right\rangle\right\}_{i=1}^{\infty}$ and $L^{\prime}=\left(\left\langle\alpha^{\prime}, t^{\prime}\right\rangle,\langle\Omega, 0\rangle\right]$. Because no countable sequence is cofinal in $\Omega_{0},\left\langle\alpha^{\prime}, t^{\prime}\right\rangle$ is not $\langle\Omega, 0\rangle$ and $L^{\prime}$ is nondegenerate. The $\bigcup_{i=1}^{\infty} O_{i}$ contains all of $L^{\prime} \times[0,1]$ except $L^{\prime} \times\left\{y_{1}\right\}$ and does not intersect $H$ so that the intersection of $H$ with $L^{\prime} \times[0,1]$ is a subset of $L^{\prime} \times\left\{y_{1}\right\}$. Since $H$ is connected, $H$ must contain $L^{\prime} \times\left\{y_{1}\right\}$ and the conclusion of the lemma follows.

Now suppose that $K=[a, b]$ is an ordered continuum and $f: K \rightarrow M$ is a continuous map such that $f(a) \in M-E_{\Omega}$ and $f(b) \in E_{\Omega} . E_{\Omega}$ is a closed subset of $M$ and there is a first point, $e$, in $K$ of $f^{-1}\left(E_{\Omega}\right)$. The map $p: M \rightarrow T$ $(T=L \times[0,1])$, defined by

$$
\begin{aligned}
& p\left(V_{\alpha, y}\right)=\langle\langle\alpha, 0\rangle, y\rangle \text { for nondegenerate elements of } M, \\
& p(\{\langle\langle\alpha, t\rangle, y, z\rangle\})=\langle\langle\alpha, t\rangle, y\rangle \text { for degenerate elements of } M,
\end{aligned}
$$

is continuous, and $p(f([a, e]))$ is a continuum in $T$ which contains only the point $p(f(e))=\left\langle\langle\Omega, 0\rangle, y_{1}\right\rangle$ of $E(E=\{\langle\Omega, 0\rangle\} \times[0,1])$ and a point $p(f(a))$ 
of $T-E$. We consider $\left\langle\alpha^{\prime}, t^{\prime}\right\rangle$ and $I_{-}^{\prime}$ from the lemma above. For the number $y_{1}$, there is at most one interval $L_{\alpha, y_{1}}$ and we consider an ordinal $\bar{\alpha}$ less than $\Omega$ and greater than both $\alpha^{\prime}$ and any $\alpha$ for which there is an $L_{\alpha, y_{1}}$. There is a first point $d$ of $[a, e]$ for which $p(f(d))=\left\langle\langle\bar{\alpha}+1,0\rangle, y_{1}\right\rangle$ and a last point $c$ of $[a, d]$ for which $p(f(c))=\left\langle\left\langle\bar{\alpha}, \frac{1}{2}\right\rangle, y_{1}\right\rangle$. Now $[c, d]$ is locally connected; hence $f([c, d])$ must be locally connected. But $f([c, d])$ must also be "the $y=y_{1}$ slice of $M$ from $\left\langle\bar{\alpha}, \frac{1}{2}\right\rangle$ to $\langle\bar{\alpha}+1,0\rangle$ " (i.e. $\{\{\langle\langle\alpha, t\rangle, y, z\rangle\} \in$ $M \mid y=y_{1}$ and $\left.\left.\left\langle\bar{\alpha}, \frac{1}{2}\right\rangle \leqq\langle\alpha, t\rangle \leqq\langle\bar{\alpha}+1,0\rangle\right\}\right)$ which is homeomorphic to the closure in the plane of the graph of $y=\sin (\pi /(1-x)), \frac{1}{2} \leqq x<1$, and is not locally connected. This involves a contradiction and the proof is complete.

\section{REFERENCES}

1. J. L. Kelley, General topology, Van Nostrand, Princeton, N.J., 1955. MR 16, 1136.

2. S. Mardešić, On the Hahn-Mazurkiewicz theorem in nonmetric spaces, Proc. Amer. Math. Soc. 11 (1960), 927-937. MR 22 \#8464.

3. - A locally connected continuum which contains no proper locally connected subcontinuum, Glasnik Mat. Ser. III 2 (22) (1967), 167-178. MR 36 \#4526.

Department of Mathematics, Iowa State University, Ames, Iowa 50010 